\section{Indoor and outdoor allergens}

Our knowledge of disease is often added to incrementally. In his editorial, Potter ${ }^{1}$ notes that two papers published in this issue of $S A M{ }^{2,3}$ add to the growing body of published information on allergens and allergies in southern Africa.

Seedat $e t$ al. ${ }^{2}$ studied indoor and outdoor allergen sensitisation in the Free State. They identified sensitisation to a previously unrecognised outdoor allergen (the spider mite, Tetranychus urticae) in the region and also found that the sensitisation rate to house-dust mites in patients with allergic rhinitis is a significant $46 \%$ for Dermatophygoides pteronyssinus and $44 \%$ for $D$. farinae. High levels of sensitisation to the spider mite have also been reported from the Hex River Valley. Since many subjects sensitised to spider mite are simultaneously polysensitised to other outdoor and indoor allergens, the clinical significance of this finding has yet to be studied, and there are no vaccines available to desensitise patients to spider mites. Allergic subjects in the Free State who had previously lived at the coast were significantly more likely to be sensitised to mites than those who had not.

Sinclair et al. ${ }^{3}$ studied house-dust mite species in Bloemfontein and unexpectedly found mites in $50 \%$ of the homes investigated. They found that in some homes high levels of mites are attained as a result of indoor microclimate factors that raise indoor humidity, such as en-suite bathrooms.

These studies suggest that the relationship between exposure, sensitisation and the expression of clinical allergic disease to house-dust mites is not a simple one and may depend not only on level of exposure but age of exposure and context of exposure.

\section{Danger of air in the wrong places}

Two short contributions graphically illustrate the potential danger of pneumopericardium and tension pneumothorax.

Sun and colleagues ${ }^{4}$ describe the successful outcome of a case of pneumopericardium resulting from a stabbed chest. Cardiac tamponade secondary to air is rare, and in adults blunt trauma is the main cause of pneumopericardium. The primary management of tension pneumopericardium is relief of the cardiac tamponade to restore haemodynamic stability. Management options include percutaneous catheter drainage, open drainage by pericardiotomy, or open drainage and repair by sternotomy/thoracotomy.

Tiemensma et al. ${ }^{5}$ report on sudden death on an aeroplane as a result of a pneumothorax. The subject had been on repeat treatment for pulmonary tuberculosis, and her medical history included drainage of pneumothoraces and pleural effusions. She experienced shortness of breath and pain soon after take-off and died shortly thereafter. A tension pneumothorax was not diagnosed despite medical practitioners being on board. The onus rests on the passenger with special needs to obtain a medical information sheet from the airline and have it completed by a medical doctor. Attending physicians are advised to inform their patients about medical conditions that may make air travel dangerous, and the authors provide the recommendations of the British Thoracic Society Standards of Care for air travel after pneumothorax.

\section{Herpes zoster ophthalmicus: Hutchinson's sign in HIV-positive patients}

Varicella zoster virus (VZV) causes two distinct viral syndromes. VZV is a common and usually benign childhood infection, manifesting as chickenpox. Herpes zoster ophthalmicus (HZO) is a potentially devastating visual disease with variable presentation caused by the re-activation of a latent infection of the trigeminal ganglion by the VZV. John Hutchinson first described the relationship between involvement of the external nasal nerve and increased likelihood of intra-ocular involvement in 1865. Van Dyk and Meyer ${ }^{6}$ report on of patients presenting with HZO.

Delays in treatment and inadequate medical therapy for herpes zoster are associated with more severe ocular complications and visual loss in HZO disease. The 6 - 15-fold increase in the incidence of $\mathrm{HZO}$ in HIV-positive patients compared with the general population suggests that HIV is the most common cause of VZV re-activation in the population at risk. Their findings confirmed that a positive Hutchinson's sign in an HIV-positive patient with $\mathrm{HZO}$ is a specific predictor of intra-ocular involvement and that $\mathrm{HZO}$ in young patients may be the presenting sign of HIV positivity. The CD4 count was not found to be a significant predictor of ocular involvement. They recommend that all patients with HZO should be seen by an ophthalmologist.

\section{Factors associated with smoking cessation}

Reductions in the prevalence of smoking in South Africa have been mainly attributed to higher cigarette prices. Ayo-Yusuf and Szymanski report on data concerning smoking from the 1998 South African Demographic and Health Survey. ${ }^{7}$ Of those who attempted to quit smoking, only $14.1 \%$ succeeded. They recommend clinical interventions, targeting alcohol-dependent smokers, and policies that boost smoke-free homes and increase knowledge of harmful effects of smoking.

\section{JPvN}

1. Potter PC. Indoor and outdoor allergens in Bloemfontein. S Afr Med J 2010; 100: 155 2. Seedat RY, Claasen J, Claasen AJ, Joubert G. Mite and cockroach sensitisation in patients with allergic rhinitis in the Free State. S Afr Med J 2010; 100: 160-163.

3. Sinclair W, Coetzee L, Joubert G. House-dust mite species in Bloemfontein, South Africa. S Afr Med J 2010; 100: 164-167.

4. Sun GR, Goosen J, Florizoone M. Cardiac tamponade secondary to tension pneumopericardium from penetrating chest trauma. S Afr Med J 2010; 100: 150

5. Tiemensma M, Buys P, Wadee SA. Sudden death on an aeroplane. S Afr Med J 2010; 100: 148149.

6. Van Dyk M, Meyer D. Hutchinson's sign as a marker of ocular involvement in HIV-positive patients with herpes zoster ophthalmicus. S Afr Med J 2010; 100: 172-174.

7. Ayo-Yusuf OA, Szymanski B. Factors associated with smoking cessation in South Africa. S Afr Med J 2010; 100: 175-180 